\title{
Introduction to the special issue: simulation, norms and laws
}

\author{
Giulia Andrighetto $\cdot$ Rosaria Conte • \\ Eunate Mayor Villalba • Giovanni Sartor
}

Published online: 7 December 2012

(C) Springer Science+Business Media Dordrecht 2012

Are we satisfied with current approaches to the study of norms? Unfortunately not, as a number of questions are still open.

First, a major dichotomy can be observed in the scientific treatment of norms. Theories of norms are grounded on two, unrelated notions, regularities and prescriptions. On the one hand, social scientists view norms as regularities of behaviour, supported by social expectations and possibly enforced through sanctions. On the other hand, philosophers of law and logicians focus on norms as prescriptions issued by definite authorities and enforced though institutional sanctions. Hence, a first set of questions pertaining to the connection between social norms and institutionalised laws comes forth. How can we distinguish normative behaviour (both social and legal) from normal conduct on the one hand and acquiescence under menace on the other? What are commonalities and differences between social conventions and institutional prescriptions? Regularities, or behavioural norms, are spontaneously emerging social phenomena, while, institutional prescriptions, are deliberately issued commands. Behavioural norms are often found either in the moral variant, as good or pro-social conduct, or in the statistical variant, as frequent, normal behaviours, while institutional prescriptions tend to collapse into legal norms, issued by specified authorities. Despite these differences it seems to us that the present gap between theories of social and institutional norms is neither desirable nor inevitable: social and institutional norms should be viewed as complementary phenomena, to be integrated in a common theoretical framework.

\footnotetext{
G. Andrighetto $\cdot$ R. Conte

LABSS/ISTC-CNR, Rome, Italy

E. M. Villalba

IRIT, Toulouse, France

G. Sartor $(\bowtie)$

Florence and Law Department-CIRSFID, European University Institute, Bologna, Italy

e-mail: giovanni.sartor@gmail.com
} 
In some contributions included in this issue, an integrated approach is indeed proposed, based on mental representations: social norms, just like legal norms, are recognized, represented and reasoned upon as prescriptions. Only a theory that explores the impact of norms on the minds of agents can explain the link between different typologies of norms.

Norms are adaptive phenomena, which respond to new problems arising in society. Hence, a second set of questions arises, i.e. how do norms emerge, change and get adapted to new circumstances? In some of the contributions presented in this issue, norms are conceptualised as social and cognitive phenomena undergoing a complex social dynamics, which allows them to emerge and change. Observable norm conformity is only the tip of the normative iceberg. The crucial dynamics lies in the minds of the agents, beneath the line of observation. Norms cannot emerge in society unless they previously get converted into mental representations. Agents abiding with norms, or violating them, act on a set of specific, norm-related, mental representations.

The mental dynamics of norms brings about a third set of questions: how should we characterize the agents among which norms emerge? Current BDI models of normative agents tackle the questions as to how people represent, reason upon, abide with or violate norms, but they fail to address an earlier problem, i.e. how can norms emerge among BDI agents.

An integrated and dynamic view of norms showing why and how to answer the three sets of questions listed above is still wanting. In particular, research in artificial intelligence and law has so far focused on the formal representation and the logics of different kinds of norms (such as norms establishing obligations, powers, sanctions, counts-as connections, etc.), and on the connection between norms and other components of legal knowledge (such as cases and concepts). Little attention has been devoted to the phenomenon of social norms, as well as to the processes of norm adoption and norm evolution.

The reader will find here no conclusive account of such phenomena. However, the main merit of this special issue is to point to agent-based simulation as an appropriate innovative methodology for the study of norms integration and dynamics, showing also to researchers in AI \& law how to complement their research and develop it in novel ways. The works presented are based on or inspired to artificial societies. Agent-based simulation is an ideal tool for exploring the twoway dynamics of norm emergence because it is relatively free of epistemological assumptions. Thus, the specific components of the different norm-like phenomenaregularities, sanctions, obligations, etc.- can be analysed and experimented upon, the complex multidirectional dynamics of norms can be explored extensively, and the relationship between cognition and social dynamics can be teased apart in a truly dynamic manner.

This special issue consists of two volumes. The first volume contains papers discussing the conceptual theoretical foundations and the methodological computational instruments for studying the dynamics of norms. In the second volume, agent-based simulations of different dynamics of norms in more or less realistic social scenarios are presented. 
In the first paper, titled "The cognitive legacy of norm simulation", Martin Neumann proposes a comparative analysis of different normative agent architectures to identify the cognitive prerequisites for the processes of norm recognition. The development of normative architectures is a burgeoning research field. However, as yet, there is no unequivocal concept for the design of normative agents.

Giulia Andrighetto and Rosaria Conte co-author the second paper, called "Cognitive Dynamics of Norm Compliance. From Norm Adoption to Flexible Automated Conformity". They propose an integrated, cognitive view of different mechanisms, reasons and pathways to norm compliance. The pragmatic modules of the normative architecture EMIL-A, i.e., the norm adoption and norm compliance modules, are presented and several alternative reasons for norm adoption and pathways to norm compliance are identified.

The third paper "Probabilistic Rule-Based Argumentation for Norm-Governed Learning Agents", co-authored by Regis Riveret, Antonino Rotolo and Giovanni Sartor, proposes a novel approach to investigate norm-governed learning agents, which combines logic-based formalism with an equation-based counterpart. The authors apply this approach to norm emergence and internalization in systems of learning agents.

The first paper of the second Volume of this special issue is titled "Identifying prohibition norms in agent societies" and is co-authored by Bastin Tony Roy Savarimuthu, Stephen Cranefield, Maryam A Purvis, and Martin K Purvis. The paper addresses the question of "how an agent identifies norms in an open agent society". To this end, it proposes an architecture enabling an autonomous agent to identify prohibition norms in a society and shows simulation data to demonstrate how this architecture works.

Sigrid Aubert and Jean-Pierre Muller co-author the second paper, called "Incorporating Institutions, Norms and Territories in a Generic Model to Simulate the Management of Renewable Resources". The paper discusses key aspects of different management options of the renewable natural resources and presents MIRANA, a computer model to simulate various scenarios of management plan implementation. The representations of institutions, norms and territories proposed by MIRANA are considered and these representations are considered in relation to the state of the art in the field of normative multi-agent systems.

The third paper, "No smoking here. Values, norms and culture in multi-agent systems" is co-authored by Francien Dechesne, Gennaro Di Tosto, Virginia Dignum and Frank Dignum. It uses the example of the introduction of the anti-smoking legislation to model the relationship between the cultural make-up, in terms of values, of societies and the acceptance of and compliance with norms. The authors present agent-based simulations to explore the effect of sanctions and their relation to values and culture.

Ulf Lotzmann, Michael Mohring and Klaus G Troitzsch co-author the fourth paper, titled "Simulating the emergence of norms in different scenarios". This paper deals with EMIL-S, a software toolbox which was designed for the simulation of processes during which norms emerged in an agent society. This toolbox implements the cognitive architecture EMIL-A, presented in the paper by Giulia Andrighetto and Rosaria Conte in this special issue. It is applied to several different scenarios, among which a micro finance scenario and a scenario modelling norm emergence in a simulated airport. 